\title{
El sistema educativo colombiano en el camino hacia la inclusión. Avances y retos ${ }^{1}$
}

\section{Yolima Ivonne Beltrán-Villamizar}

Escuela de Educación, Universidad Industrial de Santander (UIS), Bucaramanga (Colombia) atenea@uis.edu.co

\section{Ángela Sofía Vargas-Beltrán}

Grupo de Investigaciones Educativas Atenea, Bucaramanga (Colombia) sofia.vargas88@gmail.com

\section{Yexica Lizeth Martínez-Fuentes}

Grupo de Investigaciones Educativas Atenea, Bucaramanga (Colombia) yexicaml@hotmail.com

\section{Resumen}

Este trabajo busca analizar los avances y retos en materia de educación inclusiva en Colombia, a través de un análisis comparativo entre las politicas y estrategias desarrolladas en nuestro país y las medidas adoptadas por España en este mismo sentido. Para este análisis, se definieron nueve categorias a partir de los elementos clave identificados en los acuerdos internacionales precedidos por la Unesco: 1) enfoque de educación inclusiva, 2) grupos de atención prioritaria, 3) responsables y recursos, 4) estrategias de enseñanza y aprendizaje, 5) diseño curricular, 6) niveles educativos, 7) formación docente, 8) participación de la comunidad y 9) evaluación y seguimiento. Las fuentes de información corresponden a documentos oficiales de los organismos que en cada país orientan el servicio educativo, así como la legislación nacional vigente.

1 Este trabajo se deriva del proyecto de investigación denominado "Diplomado de Educación Inclusiva para población en contextos adversos" (código 8241), realizado por la Universidad Industrial de Santander en el marco del convenio de asociación número 816 de 2012 suscrito entre el Ministerio de Educación Nacional y la Universidad Industrial de Santander en representación de la Alianza para la Educación Inclusiva. 
Como resultado de este análisis, se encontró que, en materia legislativa y reconocimiento del principio de inclusión, las dos naciones de estudio no están distantes, lo que muestra la adaptación de la politica educativa colombiana a los lineamientos internacionales; sin embargo, se evidencia que la puesta en práctica de dichas orientaciones todavía dista de ser inclusiva.

\title{
Palabras clave
}

Derecho a la educación, educación integradora, educación especial, politica educacional (Fuente: Tesauro de la Unesco).

\section{The Colombian Educational System on the Road to Inclusion: Progress and Challenges}

\begin{abstract}
This paper is an attempt to analyze the progress and challenges of inclusive education in Colombia, through a comparative analysis of policies and strategies developed within the country and the measures taken by Spain in the same direction. Nine categories were defined for this analysis, based on the key elements identified in the international agreements with names preceded by "UNESCO": 1) the inclusive education approach, 2) focus groups, 3) responsible parties and resources, 4) teaching and learning strategies, 5) curriculum design, 6) educational levels, 7) teacher training, 8) community involvement and 9) evaluation and monitoring. The sources of information include official documents from the agencies in each country that oversee and direct educational services, as well as national legislation currently in effect. The results of this analysis indicate the two countries in question are not so far apart in terms of their legislation and recognition of the principle of inclusion. This shows Colombia has adapted its policy on education to reflect international guidelines. However, it is clear the implementation of these guidelines is still far from inclusive.
\end{abstract}

\section{Keywords}

Right to education, inclusive education, special education, educational policy (Source: UNESCO Thesaurus). 


\title{
O sistema educativo colombiano no caminho para a inclusão. Avanços e desafios
}

\author{
Resumo \\ Este trabalho procura analisar os avanços e os desafios em matéria de educação \\ inclusiva na Colômbia, por meio de uma análise comparativa entre as politicas e \\ estratégias desenvolvidas em nosso país e as medidas adotadas pela Espanha nesse \\ mesmo sentido. \\ Para essa análise, definiram-se nove categorias a partir dos elementos-chave identi- \\ ficados nos acordos internacionais precedidos pela Unesco: 1) enfoque de educação \\ inclusiva, 2) grupos de atenção prioritária, 3) responsáveis e recursos, 4) estratégias \\ de ensino e aprendizagem, 5) desenho curricular, 6) níveis educativos, 7) formação \\ docente, 8) participação da comunidade e 9) avaliação e seguimento. As fontes de \\ informação correspondem a documentos oficiais dos organismos que em cada país \\ orientam o serviço educativo, assim como a legislação nacional vigente. \\ Como resultado dessa análise, verificou-se que, em matéria legislativa e reconheci- \\ mento do princípio de inclusão, as duas nações de estudo não estão distantes, o que \\ mostra a adaptação da politica educativa colombiana às diretrizes internacionais; \\ no entanto, evidencia-se que a aplicação dessas orientações ainda está longe de ser \\ inclusiva.
}

\section{Palavras chave}

Direito à educação, educação integradora, educação especial, política educacional (Fonte: Tesauro da Unesco). 


\section{Introducción}

De acuerdo con algunos autores como Belth (1971) y Fernández (2013), comprender el sistema educativo de cualquier nación implica conocer el contexto social y económico en el cual se encuentra inmerso, pues los cambios en la educación siempre están acompañados de cambios políticos y económicos.

Los diferentes momentos históricos, tanto en los países desarrollados como en vía de desarrollo, han evolucionado en la percepción de la educación: esta ha pasado de ser un mecanismo para conservar los estratos sociales a ser concebida como una estrategia para garantizar la igualdad de oportunidades -independientemente de los entornos socioeconómicos de los educandos-, dejando así de ser vista como un servicio de lujo, para ser entendida como un derecho fundamental del ser humano, el cual debe ser respetado y promovido por todos los Estados (Unesco, 1948). Este trabajo busca estudiar los avances y retos en materia de educación inclusiva en Colombia, a través de un análisis comparativo entre las políticas y estrategias desarrolladas en nuestro país y en España.

A continuación abordaremos concretamente la evolución del paradigma de la educación inclusiva, partiendo desde la concepción inicial de la educación especial, hasta su concepto actual que intenta dar respuesta a las demandas sociales que abogan por la igualdad de oportunidades en los sistemas educativos, al eliminar la exclusión de las personas por su diversidad.

\section{El paradigma de la educación inclusiva}

Si bien en los años cincuenta se generó un consenso universal respecto a la necesidad de brindar educación a todas las comunidades, en la práctica se desarrollaron sistemas educativos segregacionistas, que otorgaban etiquetas a los educandos de acuerdo con sus características personales, percibiendo las diferencias de los alumnos como déficits, llegando con ello a una estigmatización entre alum- nos "normales" y "anormales". Esta segregación se visibilizó en el modelo de educación especial, el cual corresponde a la atención educativa que se presta a todos aquellos sujetos que, debido a circunstancias genéticas, familiares, orgánicas, psicológicas y sociales, son considerados excepcionales, bien en una o varias esferas concreta de su persona (intelectual, físico-sensorial, psicológica o social) (Garanto, 1984, citado por Jiménez y Vilá, 1999). Así las cosas, puede decirse que en el siglo XIX se logró el reconocimiento del derecho a la educación de todos, pero en situaciones separadas del modelo común (López, 2004).

Como respuesta a este modelo segregacionista, en los años sesenta surgieron críticas sociales hacia la marginación, la segregación, las desigualdades, etc., propiciando el debate en torno a la educación ante las diferencias (García Pastor, 1993, citado por Gómez, 2011).

Como respuesta a estos llamados sociales, a partir de los años sesenta se introdujo el concepto de necesidades educativas especiales (NEE) para reemplazar el de educación especial (EE). Este nuevo paradigma tomó fuerza a partir de 1978, con el Informe Warnock, el cual promovía la integración escolar, que buscaba las clasificaciones de minusvalía, etiquetas o categorías de las personas con discapacidad; así, el nuevo concepto de NEE asume que cada niño, independientemente de su discapacidad, tiene una necesidad particular de educación (Aguilar et al., 2010). El paradigma de la integración se soportaba en el principio de "normalización", el cual no buscaba convertir a una persona con NEE en "normal", sino aceptarla tal como es, con sus necesidades, con los mismos derechos que los demás y ofreciéndole los servicios para que pueda desarrollar al máximo sus posibilidades (Parra, 2010).

Aunque la integración constituyó un gran avance para la eliminación de la segregación en el ámbito educativo, esta tuvo un sesgo hacia el mejoramiento de la educación especial, pero no de la educación general, situación que propició un llamado entre acadé- 
micos, gobernantes y la sociedad en general, a pensar en una verdadera transformación de los sistemas educativos, con la capacidad de brindar educación de calidad y en igualdad de oportunidades a todos los estudiantes, a partir de reconocer de forma positiva las diferencias y hacer de estas una oportunidad de enriquecimiento en las aulas. Bajo estas premisas, surgió el concepto de educación inclusiva, que tomó fuerza en los años noventa, a través de la Declaración de Salamanca y Marco de Acción para las Necesidades Educativas Especiales. Tal como aseguran Echeita y Verdugo (2004), este documento es considerado el más claro e inequívoco llamado a la inclusión.

La Declaración de Salamanca ratificó el concepto de NEE haciendo hincapié en que cualquier alumno puede experimentar dificultades para aprender en diferentes momentos de su escolarización, lo cual es un proceso normal que no debe interpretarse como algo malo en el educando ni propio de unos pocos alumnos "especiales"; así se proclamó que todos los estudiantes debían tener acceso a las escuelas ordinarias (a no ser que existieran razones de peso para lo contrario), las cuales, a través de una pedagogía del niño deben satisfacer sus necesidades, y acorde con ello, se instó a los gobiernos a adoptar con carácter de ley o como política, el principio de educación integrada (Unesco, 1994).

Autores como Ainscow (2001), Arnaiz (2003), Moriña (2004), entre otros, han dado sus propios conceptos acerca de la educación inclusiva: esta, en sintesis, puede entenderse como un proceso orientado a dar respuesta a la diversidad de características y necesidades de todo el alumnado avanzando hacia una educación de calidad para todos, haciendo énfasis en el valor positivo de la diferencia (Murillo y Hernández, 2011); en la escuela inclusiva, todos los alumnos se benefician de una enseñanza adaptada a sus necesidades, y no solo los que presentan necesidades educativas especiales (Parra, 2010).

La educación inclusiva es un proceso inacabado, cuyas estrategias de acción deben ser formula- das en atención a las necesidades particulares y los contextos socioculturales de cada territorio; sin embargo, tiene un núcleo común a todos los países que es garantizar el derecho a la educación para todos. Consciente de ello, y con posterioridad a la Declaración de Salamanca, la Unesco, con participación de representantes de naciones desarrolladas y en vía de desarrollo, ha realizado en diferentes lugares del mundo encuentros que buscan socializar experiencias de prácticas inclusivas y conocer los avances y retos de diferentes Estados, lo que evidencia que se están realizando esfuerzos en todo el mundo. Estos encuentros muestran que unos países presentan mayores avances que otros, pero en general el reto de llevar los discursos de inclusión a la práctica está más vigente que nunca.

\section{Metodología}

Para analizar los avances y retos en materia de educación inclusiva en Colombia, se optó por un análisis comparativo entre las políticas y estrategias desarrolladas en nuestro país y en España. Se seleccionó España como ejemplo de comparación, por ser un país reconocido en el avance de prácticas inclusivas y en la incorporación legislativa del principio de inclusión.

La definición de las categorías se realizó a partir de los elementos clave identificados en los acuerdos internacionales precedidos por la Unesco, entre ellos la Declaración de Salamanca de 1994 y la 48 Conferencia Mundial de Educación celebrada en Ginebra en el 2008. Las fuentes de información corresponden a documentos oficiales de los organismos que en cada país orientan el servicio educativo, así como la legislación nacional vigente. A diferencia de Colombia, donde el poder legislativo es centralizado, España posee Comunidades Autónomas que, bajo unos preceptos generales, promulgan y ejecutan de forma independiente sus propias normativas; por esta razón, los aspectos que se analizan en este documento corresponden a características transversales a todo el territorio, sin detallar las particularidades de cada comunidad. 


\section{Resultados}

Colombia, al igual que la mayoría de países latinoamericanos que acogen los lineamientos orientadores de la Unesco, se ha comprometido con la transformación de su sistema educativo bajo la bandera de la inclusión. Desde la Constitución de 1991, en el artículo 67 se reconoce la educación como un derecho fundamental para todas las personas, de carácter obligatorio entre los 5 y 15 años de edad, donde las cuatro características esenciales son la disponibilidad, aceptabilidad, adaptabilidad y accesibilidad. La ley General de Educación (Ley 115 de 1994) y la Ley de Educación Superior (Ley 30 de 1992) constituyen el marco legal que orienta la prestación del servicio en todos los niveles educativos.

A pesar de que los marcos legales ya mencionados tienen implícito el principio de integración, se ha hecho necesaria la adaptación y promulgación de reglamentación complementaria que permita garantizar el pleno derecho a la educación de todos los colombianos; por ello, existen una serie de decretos y resoluciones recientes que reglamentan la prestación del servicio educativo para cada uno de los grupos identificados como población vulnerable por el mayor riesgo de exclusión en que se encuentran. Dado que la Declaración de Salamanca instó a los Gobiernos nacionales a otorgar carácter de ley la educación inclusiva, se realizó una revisión de la legislación vigente identificando la existencia de los siguientes criterios mínimos que propicien la educación inclusiva: 1) enfoque de educación inclusiva, 2) grupos de atención prioritaria, 3) responsables y recursos, 4) estrategias de enseñanza y aprendizaje, 5) diseño curricular, 6) niveles educativos, 7) formación docente, 8) participación de la comunidad, y 9) evaluación y seguimiento. Los resultados se presentan a continuación.

\section{Enfoque de educación inclusiva}

Colombia: la Ley General de Educación (Ley 115 de 1994) se refiere al principio de Integración académica y social, haciendo énfasis en personas con limi- taciones o con capacidades o talentos excepcionales. El concepto de educación inclusiva se introduce a partir del año 2006, entendido por el Ministerio de Educación Nacional (MEN) como la atención con calidad y equidad a las necesidades comunes y específicas de presentan los estudiantes (MEN, s.f.).

España: la legislación española no habla de educación inclusiva, sino de atención a la diversidad, la cual se establece como principio fundamental que debe regir toda la enseñanza básica, con el objetivo de proporcionar a todo el alumnado una educación adecuada a sus características y necesidades. La normativa general vigente en materia de educación está contenida en la Ley Orgánica 2/2006 de educación y 4/2007 universitaria.

\section{Responsables y recursos}

Colombia: en el caso de la educación básica y media, las secretarías de educación son las encargados de garantizar los procesos y los recursos para la inclusión en las instituciones públicas de sus municipios de competencia, de acuerdo con la focalización de la población vulnerable; definen las instituciones educativas que atenderán alumnos con NEE y seleccionan una entidad que realice los exámenes para determinar las condiciones de discapacidad de los alumnos (Resolución 2565 de 2003). El Decreto 366 de 2009 reglamenta la prestación de servicios de apoyo pedagógico para la atención de los estudiantes con discapacidad y con capacidades o con talentos excepcionales en el marco de la educación inclusiva, delegando esta responsabilidad a las entidades territoriales certificadas.

Las instituciones de educación superior diseñan sus propias políticas internas, orientadas a brindar atención y cobertura a las poblaciones vulnerables, y están bajo la supervisión directa del MEN a través del sistema de aseguramiento de la calidad.

España: las administraciones educativas a cargo de las comunidades autónomas son las responsables de garantizar los medios y recursos necesarios para 
que todo el alumnado alcance el máximo desarrollo personal, intelectual, social y emocional, dando cumplimiento a la legislación nacional y la normatividad propia.

La responsabilidad de las comunidades autónomas incluye los niveles de formación no universitario y universitario.

\section{Grupos de atención prioritaria}

Colombia: el MEN (2005) formuló los Lineamientos de política para la atención educativa a poblaciones vulnerables; en este documento se establecen cinco grupos poblacionales de atención prioritaria, a saber: 1) personas con discapacidad o con talentos excepcionales; 2) grupos étnicos (negros, afrocolombianos, raizales y palenqueros, indígenas y rom); 3) población víctima del conflicto armado; 4) población desmovilizada en proceso de reinserción; 5) población habitante de frontera (MEN, 2005 y 2013).

España: la LOE 2/2006, define como alumnos con necesidades especificas de apoyo educativo los siguientes: 1) alumnos con necesidades educativas especiales, entendidos como aquellos que requieran, por un periodo de su escolarización o a lo largo de toda ella, determinados apoyos y atenciones educativas específicas derivadas de discapacidad o trastornos graves de conducta; 2) alumnado con altas capacidades intelectuales; 3) alumnos con integración tardía al sistema educativo español, incluida la población inmigrante.

Normativas adicionales incluyen a la población gitana como prioritaria, como lo muestra la Estrategia Nacional para la Inclusión Social de la Población Gitana en España, 2012-2020 (Ministerio de Sanidad, Servicios Sociales e Igualdad, 2012).

\section{Estrategias de enseñanza y aprendizaje}

Colombia: el Gobierno nacional, a través del Decreto 2082 de 1996, artículos 13 y 14, establece la creación de Aulas de Apoyo Especializadas para la atención de alumnos con NEE, y Unidades de Apoyo Integral conformadas por docentes de apoyo y profesionales interdisciplinarios.

El MEN definió un Portafolio de modelos educativos flexibles para atender las necesidades de formación en diferentes contextos, que incluye las siguientes metodologías: 1) Aceleración del aprendizaje, 2) Escuela Nueva, 3) Posprimaria, 4) Telesecundaria, 5) Servicio de Educación Rural (SER), 6) Programa de Educación Continuada CAFAM, 7) Sistema de Aprendizaje Tutorial (SAT). Estos, a su vez, pueden ser adaptados de acuerdo con los diferentes contextos donde sean aplicados (MEN, s. f.).

España: para el apoyo a los centros ordinarios que atienden alumnos con NEE, existe la figura de profesor de apoyo o especializado para la atención del alumnado con necesidades educativas especiales. Si bien las funciones de este docente son establecidas de forma independiente por cada comunidad autónoma, entre sus principales funciones figura la atención directa para el desarrollo del currículo y tutorías al alumnado con NEE, apoyo a las adaptaciones curriculares y elaboración de material didáctico, entre otras (Moya, 2012).

Adicionalmente, las comunidades autónomas establecen equipos interdisciplinarios que brindan apoyo a los docentes e instituciones educativas que tienen estudiantes con necesidades de apoyo específico. Estos equipos son itinerantes en varias instituciones.

\section{Centros educativos y diseño curricular}

Colombia: el Decreto 2082 de 1996, en el artículo 17, establece que las instituciones educativas (IE) que atienden exclusivamente personas con limitaciones deben adoptar un plan gradual de adaptación de su Proyecto Educativo Institucional (PEI), bajo el principio de integración para atender todos los educandos; este plan debe ser aprobado por la 
Secretaría de Educación que corresponda. Esta medida, sumada a los colegios con aulas de apoyo especializado, representa el esfuerzo del Gobierno nacional por atender las necesidades educativas especiales eliminando el modelo segregacionista de educación especial. Acorde con lo planteado, las IE que reciban estudiantes con limitaciones, capacidades o talentos excepcionales deben especificar las adecuaciones curriculares, organizativas, pedagógicas, de recursos físicos, tecnológicos, materiales educativos, de capacitación y perfeccionamiento docente y, en general, de accesibilidad que sean necesarias.

España: existen tres tipos de centros educativos: 1) centros ordinarios de tiempo completo, donde todos los estudiantes reciben clases en una aula ordinaria de forma permanente; el proyecto curricular y la programación de aula se desarrollan de acuerdo con el nivel de enseñanza en el que se encuentra el grupo de alumnos, con medidas de adaptación curricular poco significativas y de apoyo, que cada caso requiere; 2) centros ordinarios con aulas específicas donde el alumnado que, por razón de su discapacidad, requiere una atención personalizada específica que se realiza en un aula dispuesta para tal fin en la misma institución y que, por tanto, implica un grado de integración parcial al aula ordinaria; el proyecto curricular y la programación de aula del grupo tienen un grado de adaptación significativo de acuerdo con los requerimientos; 3 ) centros de educación especial donde se escolarizan los alumnos cuyas condiciones personales de discapacidad requieran, según la evaluación psicopedagógica y el dictamen correspondiente de los equipos psicopedagógicos del sector, adaptaciones curriculares en grado extremo respecto del currículo que se desarrollaría en un centro ordinario.

\section{Niveles educativos}

Colombia: la legislación nacional prevé la educación inclusiva en todos los niveles de formación; por ello, el MEN estableció una Guía de educación inclusiva orientada principalmente a las instituciones de educación básica y media. En el año 2013, el MEN diseñó los Lineamientos de política de educación superior inclusiva, donde describe el proceso de educación inclusiva en este nivel y define unas estrategias y acciones focalizadas en los grupos poblacionales previamente definidos.

España: la Ley Orgánica 2/2006 de educación reglamenta la prestación del servicio educativo no universitario, destacando como principio la calidad, equidad y flexibilidad para la atención a la diversidad. En educación universitaria, la Ley Orgánica 4/2007 de Universidades establece que se debe garantizar el principio de igualdad de oportunidades y la no discriminación, la accesibilidad de todo el alumnado a los entornos universitarios, la inclusión en los planes de estudio de los principios de accesibilidad universal y respeto para todos, y la incorporación de ayuda personalizada, los apoyos y las adaptaciones en el régimen docente (Moriña et al., 2013).

\section{Formación docente}

Colombia: el MEN formuló, en el año 2006, el Programa de Educación Inclusiva con Calidad, en el cual se prevé una ruta metodológica para la transformación de las instituciones educativas; esta ruta está integrada por etapas, fases, estrategias y actividades adaptables a las características de cada municipio e institución. Para la puesta en marcha de esta ruta se establece la creación de un Equipo Nacional de Formadores, un Equipo de Formadores Territoriales, y un Equipo de Gestión Territorial. Estos equipos trabajan bajo un modelo de formación en cascada para orientar a las entidades territoriales y a las instituciones educativas a desarrollar el proceso de inclusión, bajo el paradigma de la inclusión y el reconocimiento de la diversidad.

Para el cumplimiento de este plan, el Gobierno nacional se comprometió con el establecimiento de políticas de formación inicial y actualización de docentes. A su vez, el Decreto 366 de 2009 encarga a las entidades territoriales certificadas el desarrollo 
de programas de formación de docentes y de otros agentes educadores para promover la inclusión de los estudiantes con discapacidad o con capacidades o talentos excepcionales en la educación formal y en el contexto social.

España: las comunidades autónomas son las encargadas de garantizar la formación permanente del profesorado no universitario; ello se realiza a través de convenios con las universidades, centros especializados de formación, agremiaciones, etc. Una de las figuras más comunes es la de Centros de Profesores, que son instituciones especializadas para la formación del profesorado, financiadas con recursos de las administraciones públicas, que ejercen en niveles educativos previos a la enseñanza universitaria, y promueven el encuentro profesional de los docentes en un marco de participación y colaboración (MECD, 2000).

\section{Participación de la comunidad}

Colombia: existe un elevado número de organizaciones sin ánimo de lucro, que promueven el respeto y cumplimiento de los derechos fundamentales en condiciones de equidad de grupos vulnerables; algunas de las más conocidas son el Instituto Nacional de Sordos (Insor), el Instituto Nacional para Ciegos (INCI), la Fundación Saldarriaga, la Asociación Nacional de Afrodescendientes Desplazados (Afrodes), y la Asociación Nacional de Síndrome de Down (Asdown), entre otras, que representan a grupos poblacionales en riesgo de exclusión.

España: actualmente existen en España 157 Comunidades de Aprendizaje constituidas en las principales zonas de exclusión social. Estas constituyen una estrategia exitosa que permite la apertura de los centros educativos a la participación de la comunidad para la transformación del territorio (Comunidades de Aprendizaje, 2014).

La Organización Nacional de Ciegos Españoles (ONCE) es una entidad sin ánimo de lucro cuya misión es facilitar y apoyar, a través de estos servicios sociales especializados, de la autonomía personal y la plena integración educativa, social y laboral de las personas con ceguera y deficiencia visual. Para ampliar la cobertura de atención, se creó la Fundación ONCE, cuya atención incluye a personas con cualquier tipo de discapacidad (ONCE, 2014).

La ONCE lidera la "Red incluD-ed" de educación inclusiva y discapacidad. El objetivo de esta red es identificar, intercambiar y difundir buenas prácticas en educación inclusiva para personas con discapacidad de toda Europa con el fin último de promover las oportunidades de empleo y la inclusión laboral. Esta fundación ha logrado el reconocimiento a nivel nacional e internacional por el apoyo a las personas ciegas o con disminución de visión; su trabajo está enlazado directamente con otras instituciones para apoyar las adaptaciones y facilitar los recursos complementarios que se requieran tanto en el ámbito educativo como laboral.

\section{Evaluación y seguimiento}

Colombia: en el año 2008, el MEN elaboró el Índice de Inclusión Educativa, el cual es una herramienta de autoevaluación de la gestión en inclusión, para el reconocimiento del estado actual de la atención a la diversidad, y el análisis de las fortalezas y oportunidades de mejoramiento para la cualificación de las condiciones de aprendizaje, participación y convivencia de la comunidad. Este índice evalúa la gestión directiva, académica, administrativa y de la comunidad.

España: el Ministerio de Educación, Cultura y Deporte presenta anualmente las estadísticas nacionales relacionadas con el alumnado con necesidad específica de apoyo educativo. Este informe incluye información de cobertura de las poblaciones focalizadas según la LOE, haciendo la distinción por centro de escolarización, necesidad específica de apoyo, comunidad autónoma y tipo de financiación.

Uno de los principales documentos guía para la autoevaluación de las instituciones educativas 
respecto a sus procesos de inclusión continúa siendo el Index para la Inclusión (Booth y Ainscow, 2002); adicionalmente, la Agencia Europea para el Desarrollo de la Educación del Alumnado con Necesidades Educativas Especiales (2009) formuló el documento Indicadores sobre educación inclusiva en Europa, el cual evalúa las áreas de Legislación, Participación y Financiación.

\section{Discusión y conclusiones}

Colombia ha avanzado en el reconocimiento de la educación inclusiva como principio infalible en la búsqueda de la educación para todos: en ese sentido, el MEN, como máximo órgano rector en materia educativa en el país, ha adelantado iniciativas en todos los niveles de formación, atendiendo las orientaciones de organismos internacionales como la Unesco. La revisión de la legislación nacional permite identificar con claridad el abordaje de los principales puntos considerados relevantes para un proceso de transformación de las instituciones educativas; sin embargo, el reto de la educación inclusiva aún está lejos de alcanzarse.

Por su condición de Estado unitario, Colombia mantiene centralizadas las funciones legislativas; sin embargo, las entidades territoriales tienen autonomía administrativa. En este sentido, las secretarías departamentales y municipales de educación adoptan un rol trascendental en la aplicación de las políticas educativas y, en este caso particular, en la puesta en marcha de las estrategias de inclusión. Con el ánimo de acoger la reglamentación nacional, las entidades territoriales y las IE públicas han iniciado los procesos de adaptación de sus proyectos educativos, de aulas de apoyo específico y de formación docente. Las instituciones ordinarias han abierto las puertas a todos los estudiantes, independientemente de su condición física, mental, social o económica, donde son los padres de familia quienes deciden el tipo de institución en la cual matriculan a sus hijos; no obstante, esta situación no constituye un avance real en materia de inclusión.
Las cifras del Departamento Administrativo Nacional de Estadística (DANE) en el año 2010 muestran que el $41 \%$ de los jóvenes de todo el país, entre los 5 y 19 años, que presentaban algún tipo de discapacidad, no estaban escolarizados; la tasa de analfabetismo en la población indígena fue de $42,6 \%$ (Centro de Estudios sobre Desarrollo Económico, 2010) y de 7,1\% para la población afrodescendiente (PNUD, 2010). Asimismo, en 2009, el MEN reportó la atención a través de servicios educativos al $43 \%$ de la población desplazada. Estas cifras evidencian que aún existen disparidades en la atención a los diferentes grupos poblacionales.

Más allá de garantizar el ingreso a las IE, el sistema educativo tiene el reto de garantizar las condiciones de permanencia de los alumnos, a través de las adaptaciones curriculares que respondan y atiendan los requerimientos de cada uno de ellos. En la práctica, estas adaptaciones no son del todo claras, situación que somete a los estudiantes a rechazo por parte de sus compañeros, condición desfavorable para el aprendizaje por entornos no adecuados a sus requerimientos, retraso en las competencias que deberían adquirir en su etapa de formación, situaciones que desembocan en deserción o cambio hacia instituciones, centros o internados que conservan el enfoque de educación especial e incluso exclusivamente terapéutico. Según reportes del MEN (2011), a nivel nacional la tasa de deserción intraanual pasó del 8,0\% en 2002 a 5,15\% en 2009; sin embargo, en zonas rurales y en algunos departamentos como Guainía, Putumayo, Vichada o Amazonas, esta tasa duplica el nivel nacional, con niveles que superan el $11 \%$. Aunque las causas de deserción son diferentes por regiones, el desplazamiento forzado es un factor que influye en cerca del $40 \%$ de los casos; de igual forma, interviene en este fenómeno la falta de pertinencia para la atención a las NEE (30\%), pedagogías inapropiadas (20\%) y las instalaciones inadecuadas de los centros educativos (15\%).

Este panorama reciente de la educación nacional muestra la necesidad de mejorar las estrategias 
de retención estudiantil, con especial atención tanto a las poblaciones vulnerables como a las zonas del país donde se focalizan y se presentan mayores riesgos de exclusión o deserción del sistema educativo: de lo contrario no se dará un efecto real de las políticas de inclusión.

A pesar de que las IE incluyeron en sus proyectos educativos el principio de inclusión, en las adaptaciones curriculares y apoyos académicos y pedagógicos continúa prevaleciendo el enfoque de discapacidades físicas y sensoriales, como en el caso de las Aulas de Apoyo Específico y los comités de apoyo departamentales, situación que evidencia una lenta incorporación de medidas efectivas para atender los demás grupos poblacionales en riesgo de exclusión. Son necesarias y urgentes las acciones que en este aspecto se adopten, pues el Estado debe dar una respuesta educativa a las víctimas del conflicto armado, así como a los grupos con diversidad étnica a través de enfoques multiculturales que permitan la formación de todos los alumnos en un mismo entorno escolar.

La revisión de las iniciativas nacionales, comparadas con un caso externo como el de España, nos muestra que en materia legislativa y reconocimiento del principio de inclusión estas dos naciones no están distantes; aun así, es de resaltar los avances en materia de participación social y comunitaria como alternativas exitosas de inclusión que vale la pena revisar en nuestro sistema. Las Comunidades de Aprendizaje constituyen una apuesta inclusiva que aportaría significativamente en el desarrollo de procesos con poblaciones vulnerables por contextos desfavorecidos, pues permiten la inclusión de toda la comunidad en torno a un ámbito de interés general como es la educación, haciendo de este un proceso participativo y contextualizado a las realidades sociales en las cuales se desarrolla. De igual forma, es necesario fortalecer las organizaciones sin ánimo de lucro, que defienden los derechos de poblaciones vulnerables; estas deben convertirse en apoyo para las instituciones educativas dado su conocimiento específico de los requerimientos de los grupos poblacionales que representan.

Al igual que con otras políticas sociales, el Estado colombiano necesita establecer un sistema riguroso de evaluación y seguimiento, que dé cuenta del avance real de las políticas y los planes diseñados: a la fecha no se ha puesto en marcha una metodología que permita monitorizar los avances y las necesidades en torno a la inclusión.

Dado el papel fundamental de los docentes en este proceso de transformación, es necesario sensibilizar tanto a los maestros en formación como a los que están en ejercicio; por tanto, se requiere replantear desde las universidades, las competencias para la atención a la diversidad que deben adquirir los estudiantes, así como la formulación de programas de formación continua que permitan la actualización de conocimiento y herramientas prácticas para la inclusión en el aula, pues gran parte de las limitaciones de esta transformación se encuentra en el desconocimiento de los docentes para abordar de manera efectiva la diversidad de los alumnos.

El camino por recorrer aún es amplio; sin embargo, el primer paso en este proceso es el reconocimiento de la inclusión como factor decisivo para el cierre de brechas sociales. El gran reto que queda por cumplir es la ejecución de las políticas planteadas, las cuales parten de la sensibilización de toda la sociedad, para la valoración de las diferencias y así atacar el principal problema que es la estigmatización, que si bien es abordado desde el ámbito educativo, no es exclusivo de ella, pues son apreciables las barreras y predisposiciones en la sociedad en general y en el mercado laboral respecto a las diferencias.

El éxito de las políticas públicas radica en la continuidad de los procesos y la capacidad de intervenir desde el origen del problema sin perder de vista el punto al que se quiere llegar. El fin de la educación inclusiva no finaliza con los procesos de aula, debe incluir un componente de sensibilización social, que permita el cambio de percepción respecto a 
las "limitaciones", para dar cabida a la concepción de inteligencias múltiples y aportes de la diversidad, solo de esta forma se logrará una adecuada vinculación y la igualdad de derechos y deberes en toda la sociedad, independientemente de las características físicas, cognitivas, racionales o culturales de la población.

\section{Referencias}

Agencia Europea para el Desarrollo de la Educación del Alumnado con Necesidades Educativas Especiales (2009). Desarrollo de indicadores sobre educación inclusiva en Europa. Verviers, Bélgica: Agencia Europea para el Desarrollo de la Educación del Alumnado con Necesidades Educativas Especiales.

Aguilar, C., Alonso, M., Padrós, M. y Pulido, M. (2010). Lectura dialógica y transformación en las Comunidades de Aprendizaje. Revista Interuniversitaria de Formación del Profesorado, 67 (24-1), 31-44. Recuperado el 5 de marzo de 2014 de: http://www.redaly c.org/articulo.oa?id=27419180003

Ainscow, M. (2001). Desarrollo de Escuelas Inclusivas: ideas, propuestas y experiencias para mejorar las instituciones escolares. Madrid: Narcea.

Arnaiz, P. (2003). Educación Inclusiva, una escuela para todos. Málaga: Archidona Aljibe.

Belth, M. (1971). La educación como disciplina científica. Buenos Aires: El Ateneo.

Booth, T. y Ainscow, M. (2002). Index for inclusion. Centre for Studies on Inclusive Education (CSIE), Bristol UK. Recuperado el 27 de febrero de 2014 de: http://www.resource-package-on-disability.org/1-one-inclusiveeduc-disability/1.3-operat-sup-materials/163-csie-index-incl-school.pdf

Centro de Estudios sobre Desarrollo Económico (2010). Encuesta longitudinal colombiana de la Universidad de los Andes. Recuperado el 5 de marzo de 2014 de: http://encuestalongitudinal.uniandes.edu.co/index.php/es/elca.

Comunidades de Aprendizaje (2014). Recuento. Recuperado el 27 de febrero de 2014 de: http://utopiadream. info/ca/?page_id $=1645$

Decreto 2082 de 1996 [Presidencia de la República de Colombia]. Por el cual se reglamenta la atención educativa para personas con limitaciones o con capacidades o talentos excepcionales. Noviembre 18 de 1996.

Decreto 366 de 2009 [Presidencia de la República de Colombia]. Por medio del cual se reglamenta la organización del servicio de apoyo pedagógico para la atención de los estudiantes con discapacidad y con capacidades o con talentos excepcionales en el marco de la educación inclusiva. Febrero 9 de 2009.

Departamento Nacional de Estadística (2010). Población con registro para la localización y caracterización de las personas con discapacidad. Recuperado el 3 de marzo de 2014 de: https://www.dane.gov.co/index. php/poblacion-y-registros-vitales/discapacidad 
Echeita, G.y Verdugo, M. (2004). La Declaración de Salamanca sobre Necesidades Educativas Especiales 10 años después. Valoración y Prospectiva. Salamanca: Instituto Universitario de Integración en la Comunidad. Recuperado el 3 de marzo de 2014 de: http://sid.usal.es/idocs/F8/FDO9045/declaracion_salamanca_completo.pdf

Fernández, M. (2013). Análisis del contexto educativo en el contexto social. En Carrasco, M., Coronel, J., Fernández, S., García, M., González, S.y Moreno, E. (2013). Conocer y comprender las organizaciones educativas: una mirada a las cajas chinas (pp.42-55). Madrid: Ediciones Pirámide.

Gómez, I. (2011). Dirección escolar y atención a la diversidad: rutas para el desarrollo de una escuela para todos. Tesis inédita de doctorado. Universidad de Huelva, Departamento de Educación, Huelva, España.

Jiménez, P.y Vila, M. (1999). De educación especial a educación en la diversidad. Málaga: Ediciones Aljibe.

Ley 30 de 1992 [Congreso de la República de Colombia]. Por el cual se organiza el servicio público de la Educación Superior. Diciembre 28 de 1992.

Ley 115 de 1994 [Congreso de la República de Colombia]. Por la cual se expide la ley general de educación. Febrero 8 de 1994.

Ley 2/2006 [Jefatura del Estado de España]. Ley Orgánica 2/2006 de Educación.

Ley 4/2007 [Jefatura del Estado de España]. Ley Orgánica 4/2007 por la que se modifica la Ley Orgánica 6/2001, de 21 de diciembre, de Universidades. Abril 12 de 2007.

López, M. (2004). Construyendo una escuela sin exclusiones. Una forma de trabajar en el aula con proyectos de investigación. Málaga: Ediciones Aljibe.

Ministerio de Educación, Cultura y Deporte (2000). El sistema educativo Español 2000. Madrid: Faresa.

Ministerio de Educación Nacional (s.f.). Portafolio de Modelos Educativos. Dirección de Poblaciones y Proyectos Intersectoriales. Bogotá. Recuperado el 3 de marzo de 2014 de: http://www.oei.es/quipu/colombia/ portafolio_modelos_educ.pdf

Ministerio de Educación Nacional (2005). Lineamientos de política para la atención educativa a poblaciones vulnerables. Bogotá. Recuperado el 3 de marzo de 2014 de: http://www.mineducacion.gov.co/cvn/1665/ articles-90668_archivo_pdf.pdf

Ministerio de Educación Nacional (2008). Guía para el mejoramiento institucional. De la autoevaluación al plan de mejoramiento. Serie guías, 34. Bogotá. Recuperado el 5 de marzo de: http://www.mineducacion. gov.co/1621/articles-177745_archivo_pdf.pdf

Ministerio de Educación Nacional (2009). Encuesta Nacional de Deserción Escolar (ENDE). Bogotá. Recuperado el 5 de marzo de 2014 de: http://www.mineducacion.gov.co/1621/articles-293672_archivo_pdf_presentacion.pdf

Ministerio de Educación Nacional (2009). Plan de Cobertura Acceso y Permanencia. Bogotá. Recuperado el 5 de marzo de 2014 de: http://www.mineducacion.gov.co/1621/articles-183110_archivo_pdf2.pdf 
Ministerio de Educación Nacional (2013). Lineamientos política de educación superior inclusiva. Bogotá. Recuperado el 28 de febrero de 2014 de: http://www.dialogoeducacionsuperior.edu.co/1750/articles-327647_ documento_tres.pdf

Ministerio de Sanidad, Servicios Sociales e Igualdad (2012). Estrategia Nacional para la Inclusión Social de la Población Gitana en España 20122020. Madrid. Recuperado el 4 de marzo de 2014 de: https://www.msssi. gob.es//ssi/familiasInfancia/inclusionSocial/poblacionGitana/docs/EstrategiaNacionalEs.pdf

Moriña, A. (2004). Teoría y práctica de la educación inclusiva. Archidona: Aljibe

Moriña, A., López, R., Melero, N., Cortés, M. y Molina, D. (2013). El profesorado en la universidad ante el alumnado con discapacidad: itendiendo puentes o levantando muros? Revista de Docencia Universitaria, 11 (3), 423-442.

Moya, A. (2012). El profesorado de apoyo en los centros ordinarios. Nuevas funciones, nuevas contradicciones. Educatio Siglo XXI, 30 (1), 71-88. Recuperado el 4 de marzo de 2014 de: http://revistas.um.es/educatio/article/viewFile/149141/132131

Murillo, J. y Hernández, R. (2011). Una dirección escolar comprometida con la justicia social. Observatorio Social de la Educación, 4, 19-28.

ONCE (2014). Qué es la ONCE. Recuperado el 3 de marzo de 2014: http://www.once.es/new/que-es-la-ONCE

Parra, C. (2010). Educación inclusiva: un modelo de educación para todos. Revista ISEES (8), 73-84. Recuperado el 28 de febrero de 2014 de: http://www.isees.org/file.aspx?id=7090

Programa de las Naciones Unidas para el Desarrollo (2010). Situación socioeconómica de la población afrocolombiana en el marco de los Objetivos de Desarrollo del Milenio. Panamá. Recuperado el 4 de marzo de 2014 de: http://www.afrodescendientes-undp.org/FCKeditor_files/File/AFRO_COLOMBIA.pdf

Resolución 2565 de 2003. [Ministerio de Educación Nacional]. Por la cual se establecen parámetros y criterios para la prestación del servicio educativo a la población con necesidades educativas especiales. Octubre 24 de 2003.

Unesco (1948). Declaración universal de los derechos humanos. Recuperado el 4 de marzo de 2014 de: http:// www.un.org/es/documents/udhr/

Unesco (1994). Declaración de Salamanca y Marco de Acción para las Necesidades Educativas Especiales. Salamanca. Recuperado el 5 de marzo de 2014 de: http://www.unesco.org/education/pdf/SALAMA_S.PDF

Unesco (2008). Conferencia Internacional de Educación. Cuadragésima octava reunión. Ginebra. Recuperado el 4 de marzo de 2014 de: http://www.ibe.unesco.org/fileadmin/user_upload/Policy_Dialogue/48th_ICE/ICE_ FINAL_REPORT_spa.pdf 